\title{
Association of high-sensitivity cardiac troponin $T$ concentrations with the left ventricular global function index after ST-segment elevation myocardial infarction
}

\author{
Hans-Josef Feistritzer ${ }^{1 *}$, Sebastian J Reinstadler', Gert Klug ${ }^{1}$, Marie-Therese Gröber ${ }^{1}$, Johannes M Mair ${ }^{1}$, \\ Michael Schocke ${ }^{2}$, Wolfgang-Michael Franz ${ }^{1}$, Bernhard Metzler ${ }^{1}$
}

From 18th Annual SCMR Scientific Sessions

Nice, France. 4-7 February 2015

\section{Background}

The left ventricular global function index (LVGFI) is a novel indicator of cardiac performance. Recently, the LVGFI was linked to left ventricular ejection fraction and infarct characteristics in patients presenting with acute ST-segment elevation myocardial infarction (STEMI). In this study we sought to investigate the association of serially measured high-sensitivity cardiac troponin $\mathrm{T}$ (hs-TnT) concentrations and LVGFI assessed early after acute STEMI.

\section{Methods}

122 patients with first STEMI (mean age $58 \pm 11$ years) reperfused by primary percutaneous coronary intervention (p-PCI) were enrolled in this observational study. All patients underwent cardiac magnetic resonance (CMR) imaging within the first week after STEMI. Left ventricular dimensions and function were measured by cine trueFISP sequences. hs-TnT concentrations were assessed serially for up to 48 hours after p-PCI by a commercially available immunoassay. Fisher's $\mathrm{r}$ to $\mathrm{z}$ transformation was applied for comparison of correlation coefficients.

\section{Results}

The median delay to reperfusion was $205 \mathrm{~min}$ (IQR 144 to $391 \mathrm{~min})$. The mean LVGFI was $35 \pm 8 \%$. The median value of maximum hs-TnT concentrations was $5043 \mathrm{ng} / \mathrm{l}$ (IQR 2322 to $8104 \mathrm{ng} / \mathrm{l}$ ). The LVGFI was significantly

'University Clinic of Internal Medicine III, Cardiology and Angiology, Medical University of Innsbruck, Innsbruck, Austria

Full list of author information is available at the end of the article associated with maximum creatine kinase activity $(\mathrm{r}=$ -0.473, $\mathrm{p}<0.001$ ) and with maximum hs-TnT concentration $(r=-0.478, p<0.001)$. Statistically significant correlations were also detected between LVGFI and single time-point hs-TnT concentrations (all $\mathrm{r}<-0.422$, all $\mathrm{p}<$ 0.001). Receiver operator characteristics analysis (area under the curve: $0.85,95 \%$ CI 0.75 to 0.95 ) revealed hsTnT concentrations measured 6 hours after p-PCI to most accurately predict a LVGFI below the median value of $34 \%$.

\section{Conclusions}

This study demonstrates that hs-TnT concentrations assessed serially after acute STEMI are associated with LVGFI. hs-TnT levels measured 6 hours after p-PCI best predicted a LVGFI below the median value.

\section{Funding}

Austrian Society of Cardiology.

\section{Authors' details}

${ }^{1}$ University Clinic of Internal Medicine III, Cardiology and Angiology, Medical University of Innsbruck, Innsbruck, Austria. ${ }^{2}$ Departement of Radiology I, Medical University of Innsbruck, Innsbruck, Austria.

Published: 3 February 2015

doi:10.1186/1532-429X-17-S1-P134

Cite this article as: Feistritzer et al:: Association of high-sensitivity cardiac troponin T concentrations with the left ventricular global function index after ST-segment elevation myocardial infarction. Journal of Cardiovascular Magnetic Resonance 2015 17(Suppl 1):P134. 